\title{
Xerostomía postradioterapia. Eficacia de tratamientos tópicos basados en aceite de oliva, betaína y xilitol
}

\section{Xerostomia post-radiation therapy. Efficacy of topical treatments based on olive oil, betaine and xylitol}

\author{
Martín Martín M*, López M*, Cerezo L*
}

\section{RESUMEN}

La xerostomía es la complicación más frecuente en los pacientes que reciben radioterapia para el cáncer oral. La reducción en la tasa de flujo salival y una disminución de su pH están directamente relacionado con un cambio en la composición de la saliva y el desplazamiento de la microflora oral hacia especies bacterianas cariogénicas. Por otro lado sabemos que la xerostomía se asocia a dificultad para el habla, la masticación, la deglución, cambios en el sabor, caries dentales, sensación de ardor, infecciones microbianas y un deterioro de la calidad de vida.

El manejo de la xerostomía incluye la prevención, la estimulación y el tratamiento sintomático. La prevención no siempre es posible a pesar de que con radioterapia de intensidad modulada (IMRT), se puede administrar dosis más bajas de radiación a las glándulas parótidas. Los fármacos estimuladores, como los agonistas colinérgicos, han demostrado mejorar la xerostomía, sin embargo tienen efectos secundarios y están contraindicados en algunas patologías. Hoy disponemos de una gran variedad de productos tópicos para el alivio de los síntomas de la boca seca, como la goma de mascar, pastillas sin azúcar, sustitutos salivales, cremas hidratantes o pastas dentales.

Un trabajo previo nos indica que el uso diario de productos de boca seca tópicos que contienen aceite de oliva, betaína y xilitol es seguro y eficaz en el alivio de los síntomas de xerostomía en pacientes con xerostomía inducida por fármacos. En este trabajo se revisa la eficacia de las diversas opciones de tratamiento para la hiposalivación inducida por la radiación y presentamos nuestros resultados con el uso de productos tópicos específicos en estos pacientes.

Palabras clave: Radioterapia, xerostomía, calidad de vida, cuestionarios, escala analógica visual, neoplasia de cabeza y cuello.

\section{SUMMARY}

Xerostomia is the most frequent complication among patients who receive radiotherapy for oral cancers. A reduction in salivary flow rate and decrease of its $\mathrm{pH}$ is paralleled with a change in saliva competence and shifting of oral microflora to cariogenic bacterial spices. Therefore difficulties in speech, mastication, swallowing, changes in taste, dental caries, burning sensation, microbial infections and a compromised quality of life are associated with the presence of xerostomia.

Studies have led to three therapeutic approach for xerostomia treatment: prevention, stimulation and symptomatic treatment. Prevention it is not always possible, although intensity modulated radiation therapy (IMRT) technique, gives the ability to delivering lower doses of radiation to parotid glands. Stimulation agents as cholinergic agonist have all demonstrated some ability to improve xerostomia, however have side effects and are contraindicated for certain medical disorders. There are a huge variety of products for relief dry mouth symptoms as Chewing gum, sugarfree lozenges, salivary substitutes and moisturizers, toothpastes. 
A previous study report that the daily use of topical dry mouth products containing olive oil, betaine and xilitol is safe and effective in relieving symptoms of xerostomia in a population with polypharmacy-induced xerostomia. The objective of this study is to review efficacy of various treatment options for radiation-induced hyposalivation and present our results with the use of specific topical products in this patients.

Key words: Radiotherapy, xerostomia, quality of life, questionnaries, visual analogic scale, head and neck neoplasms.

Fecha de recepción: 15 de enero de 2014.

Aceptado para publicación: 19 de marzo de 2014.

Martín Martín M, López M, Cerezo L. Xerostomía postradioterapia. Eficacia de tratamientos tópicos basados en aceite de oliva, betaína y xilitol. Av. Odontoestomatol 2014; 30 (3): 161-170.

\section{INTRODUCCIÓN}

La saliva es un fluido viscoso claro y acuoso, secretado por las glándulas salivares mayores: glándula parótida, submaxilar, sublingual y las glándulas salivares menores distribuidas a lo largo de toda la mucosa oral. Contiene dos tipos principales de secreciones una serosa y otra mucosa. La secreción serosa está constituida por enzimas digestivas, se produce fundamentalmente por la parótida bajo estímulos como la masticación, el roce de la mucosa, olores, etc. y favorece la deglución. La secreción mucosa es rica en agentes lubricantes y mucina. Está producida el $90 \%$ por las glándulas submaxilares y el $10 \%$ restante, por la sublingual y las glándulas salivares menores, en condiciones de no estimulación. Favorece la lubricación y humidificación de la mucosa oral. Tiene además un alto contenido en agentes antimicrobianos como lisozimas, inmunoglobulinas etc. Todo ello hace que la xerostomía, definida como una disminución o ausencia de flujo salivar, ocasione una serie de problemas como:

- Dificultad para el habla produciendo disartria.

- Dolor o molestias orales, como boca ardiente.

- Dificultad para la masticación, la deglución y la percepción de los sabores.

- Favorece la aparición de infecciones, especialmente fúngicas, por la formación de fisuras y ulceraciones en la mucosa y la aparición de caries dental.

- Afecta el sueño porque la sequedad despierta al paciente en repetidas ocasiones durante la noche.

Son muchas las causas que pueden producir xerostomía, aparece en enfermedades sistémicas como el síndrome de Sjögren, y es un efecto secundario de múltiples fármacos y tratamientos oncológicos como la irradiación.

\section{XEROSTOMÍA POSTRADIOTERAPIA}

La xerostomía es una complicación frecuente después de la irradiación de un tumor de cabeza y cuello. Dependiendo de la dosis que reciba la glándula parótida el flujo salivar puede disminuir entre un 50-60\% del basal, después 10-16 Gray (Gy) y un 20\% del normal después de 5-6 semanas de tratamiento. Dosis medias de 26 Gy sobre ambas parótidas disminuyen el flujo salivar estimulado (1). Muy importante también, en la aparición de síntomas de xerostomía, es la dosis de irradiación en glándulas submaxilares, por su importante contribución al flujo salivar no estimulado y en la producción de mucina. Se ha visto que dosis por encima de 39 Gy sobre dichas glándulas disminuye el flujo salivar no estimulado de forma exponencial un 3\% por cada Gy y se recupera si la dosis media no supera los 39 Gy (2).

Después de radioterapia se producen una serie de cambios histológicos tardíos en el parénquima de las glándulas salivares, como un aumento del espacio extracelular extravascular, y una disminución de la permeabilidad vascular. Se pierden las células acinares por la muerte de las células troncales, y aparece fibrosis intersticial por aumento de los depósitos de fibras de colágeno. Otros fenómenos documentados son: proliferación de los ductos, pérdida de los gránulos secretores e infiltración de células inflamatorias como linfocitos y células plasmáticas. Estos cambios son más prominentes en las glándulas submaxi- 
lares que en las parótidas (3). Tras la irradiación se produce no solo cambios en el volumen de saliva sino también en su composición, se disminuye la actividad de la amilasa y del bicarbonato con el consiguiente aumento de $\mathrm{pH}$. Se produce también un aumento de la viscosidad de la osmolaridad, y aumento la concentración de lactoferrina, sodio y cloro (4).

\section{EVALUACIÓN DE LA XEROSTOMÍA}

La cuantificación de flujo salivar es la forma utilizada con mayor frecuencia para conocer la función de las glándulas salivares. En general, el flujo salivar se mide durante 5 minutos y se determina el flujo salivar en $\mathrm{ml}$ min-1. Se puede medir el flujo no estimulado y el estimulado, el global o el de una glándula en concreto mediante la cateterización del conducto salivar requerido. Se considera normal un flujo salivar por encima de 0,25 ml min-1, la gravedad de la disminución de flujo salivar se refleja en la Tabla 1.

Las pruebas de imagen no se utilizan de rutina en la clínica para evaluar la xerostomía:

- Xialografía o escintigrafía muestran cambios similares post RT y s. Sjögren, se puede ver dilatación por estenosis y obstrucción de los ductos. Son difíciles de realizar, requieren colaboración del paciente.

- La ecografía de una glándula irradiada también es similar al de una parótida de un paciente con síndrome de Sjögren, apreciándose ecoestructura heterogénea y múltiples áreas hipoecoicas. No permite ver el lóbulo profundo de la parótida por la interferencia de la rama mandibular porque lo que no se puede medir el tamaño, y puede haber una gran variabilidad en la apreciación de la glándula según el radiólogo.

\begin{tabular}{|c|c|}
\hline \multicolumn{2}{|c|}{ TABLA 1.- DATOS REFERENTES AL FLUJO } \\
SALIVAL NORMAL
\end{tabular}

- La tomografía computerizada permite apreciar aumento de densidad de la glándula pero es muy inespecífica, requiere contraste y aporta poca información.

- La resonancia nuclear magnética puede mostrar cambios en las glándulas salivares y en los ductos, tras la irradiación pero tiene limitaciones para visualizar las ramas pequeñas de los ductos salivares, es cara, y se artefacta con implantes metálicos que con frecuencia portan los pacientes en los dientes.

Más útiles en la clínica son las escalas de valoración de calidad de vida y visual analógica de las que hablaremos más adelante.

\section{TRATAMIENTO DE LA XEROSTOMÍA POSTRADIOTERAPIA}

\section{Prevención}

Es importante evitar, en lo posible, el daño sobre las glándulas salivares.

Las opciones para proteger las glándulas salivares durante la irradiación actualmente son:

A) Minimizar las dosis de irradiación: Las nuevas técnicas de irradiación como la radioterapia con modulación de intensidad (IMRT) permiten disminuir la dosis de irradiación en las glándulas salivares. El objetivo es conseguir dosis media en parótidas menores de 26 Gy en parótidas y 20 Gy en glándulas submandibulares. Disminuyendo la dosis de irradiación mediante IMRT, sobre parótidas numerosos estudios han descrito reducción en la incidencia de xerostomía grado 3, que puede llegar a ser de un $67 \%$ con técnicas 3D convencionales a un $12 \%$ con IMRT (5).

B) Quirúrgicas: Otra forma de proteger las glándulas salivares es la trasposición quirúrgica antes de la irradiación. En general se realiza una trasposición de la glándula submandibular contralateral al tumor a la región submental. Esta técnica puede mantener niveles de saliva similares a los previos a la irradiación (6). Sin embargo es una técnica quirúrgica complicada, puede retrasar los tratamientos oncológicos y no siempre es posible preservar de la irradiación la región submentoniana. 
C) Farmacológicas: Se han estudiado múltiples fármacos con intención radioprotectora. De ellos, el que se ha mostrado más efectivo es la Amifostina. Se trata de una sal fosfórica que se absorbe fundamentalmente en los tejidos normales por la mayor concentración de fosfatasa alcalina y elimina los radicales libres protegiendo a la membrana y el DNA, del daño, producido por estos. En estudios aleatorizados, ha demostrado disminuir la xerostomía mayor de grado 2 cuando se utiliza RT exclusiva en pacientes tratados con radioquimioterapia que, actualmente, es el tipo de tratamiento más utilizado; los estudios son contradictorios $(7,8)$. Este hecho, junto con los efectos secundarios que produce, hipotensión y vómitos, así como la necesidad de administrarla de forma intravenosa, hacen que su utilización sea muy limitada.

Palifermin es un factor de crecimiento de los queratinocitos; ha demostrado disminuir la xerostomía cuando se utiliza radioterapia con hiperfraccionamiento, esto es, menos dosis de irradiación por sesión pero más fracciones y más dosis final pero no con fraccionamientos convencionales (9).

\section{Tratamiento farmacológico}

Una vez que la xerostomía está establecida, la pilocarpina ha demostrado aumentar la saliva en pacientes irradiados con dosis de 5 mg/8 h (10). Las guías de Ontario para el uso de Pilocarpina en pacientes irradiados contemplan que: los pacientes deben tener evidencia de función salivar previa y no contraindicación médica. La duración del tratamiento no está determinada, no hay evidencia más allá de 3 meses.

Cevimelina, a dosis de $30 \mathrm{mg} / 8 \mathrm{~h}$, a pesar de aumentar la saliva no estimulada no mejora la salud bucal ni la calidad de vida (11).

\section{Los autocuidados}

Los autocuidados que se recomiendan en pacientes con xerostomía de cualquier origen son:

- Visitas periódicas al dentista: retirar placa, tratamiento. de infecciones gingivales, tratamiento precoz de la caries.
- Dentífrico fluorado frecuente.

- Evitar productos que contengan lauril sulfato sódico.

- Evitar alimentos con azúcares, picantes, astringentes, muy calientes y fríos. Eliminar tabaco y alcohol.

- Utilizar saliva artificial o ingesta frecuente de agua.

- Utilizar estimulantes de saliva: cítricos, caramelos o chicles sin azúcar.

- Las dentaduras postizas se deben retirar durante la noche, mantenerlas limpias: las acrílicas en hipoclorito de sodio, metálicas en clorhexidina.

La realización de estos cuidados debería mejorar la salud bucal y la calidad de vida de los pacientes pero hay pocos estudios realizados. En una revisión sobre medidas no farmacológicas en xerostomía Cochrane (12) concluyen que:

- Como sustituto salivar el aerosol de triester de glicerol oxigenado es más eficaz que el aerosol electrolítico con agua.

- Masticar chicle aumenta la saliva pero no hay evidencia que sea mejor que el sustituto salivar.

- Geles, reservorios salivares y sistemas de atención bucal pueden ser beneficiosos pero son necesarios más estudios para comprobarlo.

\section{XEROSTOM ${ }^{\circledR}$ en la xerostomía postirradiación}

Dada escasez de estudios sobre el tratamiento tópico en xerostomía postradioterapia, nos propusimos realizar un estudio con productos basados en aceite de oliva, betaína y xilitol, de la marca comercial Xerostom ${ }^{\circledR}$. El uso de forma reglada de estos productos, en pacientes con xerostomía de causa farmacológica, ha demostrado que, mejoran la mayoría de los síntomas producidos por la xerostomía y son capaces de aumentar el flujo salivar (13).

Los componentes fundamentales de los productos Xerostom ${ }^{\circledR}$ son:

- Aceite de oliva: Lubricante, disminuye los efectos en la mucosa producidos por la falta de saliva. Además inhibe el crecimiento bacteriano (14).

- Betaína: Mantiene la humedad, les protege de irritaciones (15).

- Xilitol: Tiene actividad anticaries (16). 
- Vit E: Reduce la irritación de la mucosa.

- Flúor y calcio.

- Alantoína: Evitar fisuras.

En base a todo lo anterior, los objetivos del estudio son:

1. Principal: Determinar el impacto en calidad de vida y evaluar la disminución de síntomas provocados por xerostomía postradioterapia con la aplicación tópica de productos basados en aceite de oliva betaína y xilitol.

2. Secundario: Determinar si hay incremento del flujo salivar tras la aplicación tópica de dichos productos durante 15 días.

\section{MATERIAL Y MÉTODOS}

Se incluyeron en el estudio 40 pacientes que habían recibido tratamiento radioterápico por algún tumor de cabeza y cuello y se presentaba algún síntoma de boca seca. Los pacientes fueron instruidos para la utilización del dentífrico 3 veces al día después de las comidas principales, utilizar el gel alrededor de las encías por las noche, enjuagues con colutorio y spray como sustituto salivar, todas las veces que deseasen pero al menos 8 veces entre todos los productos.

Los criterios de inclusión en el estudio fueron:

- Pacientes mayores de 18 años que hubieran recibido tratamiento radioterápico por un carcinoma de cabeza y cuello.

- Que presentaran síntomas de boca seca.

- Capaces de utilizar de forma correcta colutorio, gel oral y dentífrico durante el periodo de estudio.

- Capaces de contestar los cuestionarios de calidad de vida en EVA.

- Que firmaran el consentimiento informado.

Los criterios de exclusión:

- Pacientes con síndrome de Sjögren.

- Incapaces de administrarse los productos a estudio, y/o incapaces de comprender el consentimiento informado.

- Pacientes que hubieran tomado Pilocarpina en los 7 días previos a entrar en el estudio.

- Pacientes que tuvieran previsto una cirugía oral durante el proceso de estudio.
- Pacientes con patologías médicas asociadas que requirieran cambios en la medicación de base.

- Pacientes que fueran ingresados por algún problema médico durante el estudio serian excluidos.

\section{Medidas basales}

\section{Flujo salivar}

Para calcular el flujo salivar se realizó una recolección de la saliva no estimulada, mediante emisión directa a un vaso de plástico que era pesado en una balanza de alta precisión. No se permitía a los pacientes comer y beber nada en las dos horas previas a la recolección de saliva. También se indicó a los pacientes que no debían lavarse los dientes, fumar o masticar chicle o caramelos antes de esta medición del flujo salivar.

\section{Cuestionario de calidad de vida}

Se utilizó un cuestionario de calidad de vida de 15 preguntas. Las preguntas de este cuestionario se refieren a cómo afecta la boca seca a la calidad de vida en cuatro aspectos fundamentales:

- Función física:

1. La sequedad de boca limita la cantidad de comida que como.

6. La sequedad de boca me dificulta el hablar delante de otras personas.

10. La sequedad de boca interfiere con mis actividades diarias.

12. La sequedad de boca dificulta saborear la comida.

- Dolor:

2. La sequedad de boca me resulta incómodo/a.

3. La sequedad de boca me preocupa.

7. La sequedad de boca me pone nervioso/a.

9. La sequedad de boca no me deja disfrutar de la vida.

- Función personal/psicológica:

8. La sequedad de boca hace que me preocupe por el aspecto de mis dientes y boca.

13. La sequedad de boca reduce mi felicidad en general.

14. La sequedad de boca afecta todos los aspectos de mi vida. 
15. Si tuviera que pasar el resto de mi vida con la sequedad de boca actual me sentiría.

- Función social:

4. La sequedad de boca me mantiene alejado de los demás.

5. La sequedad de boca me incomoda cuando como delante de otras personas.

11. La sequedad de boca interfiere en mis relaciones íntimas.

Cada pregunta tiene 4 posibles respuestas: en absoluto, algo, bastante, mucho, muchísimo.

\section{Cuestionario escala visual analógica (EVA)}

Este cuestionario, validado en distintos estudios (17), consta de 8 preguntas referentes a los síntomas que provocan la boca seca sobre función, cantidad de saliva, y grado sed. El grado de molestia en cada pregunta se traslada a una línea de $100 \mathrm{~mm}$, siendo 0 la menor molestia y 100 la mayor. Las preguntas de este cuestionario son:

1. Valore la dificultad que sientes al hablar debido a la sequedad de boca.

2. Valore la dificultad que sientes al tragar debido a la sequedad de boca.

3. Valore cuanta saliva tienes en la boca.

4. Valore la sequedad de boca.

5. Valore la sequedad de garganta.

6. Valore la sequedad de labios.

7. Valore la sequedad de lengua.

8. Valore el nivel de sed.

\section{Análisis estadístico}

Los datos fueron analizados con el paquete SPSSv16. Se realizó un estudio descriptivo de medias, medianas y porcentajes de las características demográficas y de las escalas anteriormente referidas así como del flujo salivar. Para la comparación de valores antes y después de la utilización de los productos se utilizó análisis t pareados.

Como se dijo anteriormente, la encuesta de calidad de vida evalúa 4 dominios; se hizo una media de las puntuaciones para cada dominio así como una media total de los cuatro dominios. Los cambios en el tiempo de las encuestas de calidad de vida fueron analizados con varianza de medidas repetidas. La variable independiente fue el tiempo y las dependientes la media global de los cuatro dominios y las medias de cada dominio.

\section{Resultados}

Se reclutó a 40 pacientes, algunos de ellos fueron llamados por teléfono, a otros se les invitó a participar en el estudio cuando vinieron a consulta de revisión programada. De ellos, 25 eran hombres y 15 mujeres; la edad media fue de 65 años. La localización tumoral más frecuente fue la laringe. Además del tratamiento radioterápico, 25 pacientes tomaban algún medicamento que podía causar como efecto secundario sequedad de boca. Hasta 20 pacientes no presentaba ningún flujo salivar no estimulado. Las características de los pacientes pueden verse en la Tabla 2.

Treinta y ocho pacientes completaron el estudio, 1 de ellos con afagia completa y portador de sonda

\begin{tabular}{|l|c|}
\hline \multicolumn{2}{|c|}{ TABLA 2.- DATOS REFERENTES A LA } \\
MUESTRA \\
\hline Características & N (\%; max-min) \\
\hline - Sexo: & $25(62,5 \%)$ \\
- Hombre & $15(37,5 \%)$ \\
- Mujer & $65(47-99)$ \\
- Edad & \\
- Localización tumoral: & $12(30 \%)$ \\
• Faringe & $14(35 \%)$ \\
- Laringe & $12(30 \%)$ \\
- Cavidad Oral & $2(5 \%)$ \\
- Otras & \\
- No Fármacos xerostomía: & $15(37 \%)$ \\
• 0 & $9(22,5 \%)$ \\
- 1 & $12(30 \%)$ \\
• 2 & $3(2,5 \%)$ \\
- 3 & $1(2,5 \%)$ \\
- 5 & $3(1 \mathrm{mes}-9$ años) \\
- Tiempo desde fin RT
\end{tabular}


nasogástrica, refirió en la segunda visita que tras tres días de utilizar los productos tuvo que dejarlos porque le produjeron mucha saliva que no podía tragar y le resultaba muy incomodo limpiarse continuamente. Otro paciente no completó las dos semanas de utilización de los productos porque no le parecieron mejores de los que utilizaba previamente. Las respuestas de ambos pacientes en la segunda visita formaron parte del análisis.

\section{Calidad de vida}

Hubo mejoría en 14 de las 15 preguntas de la encuesta de calidad de vida tras la utilización de los productos Xerostom ${ }^{\circledR}, 7$ de ellas con diferencias estadísticamente significativas (Figura 1). Las cuestiones que mejoraron fueron:

1. La sequedad de boca limita la cantidad de comida que como (p: 0,05).

2. La sequedad de boca me resulta incómodo/a (p: $0,0)$.

3. La sequedad de boca me preocupa $(0,01)$.

5. La sequedad de boca me incomoda cuando como delante de otras personas $(0,03)$.

6. La sequedad de boca me dificulta el hablar delante de otras personas $(0,006)$.

7. La sequedad de boca me pone nervioso/a $(0,006)$.

12. La sequedad de boca dificulta saborear la comida $(0,01)$.

También hubo diferencias estadísticamente significativas en los 4 aspectos a los que hacían referencia

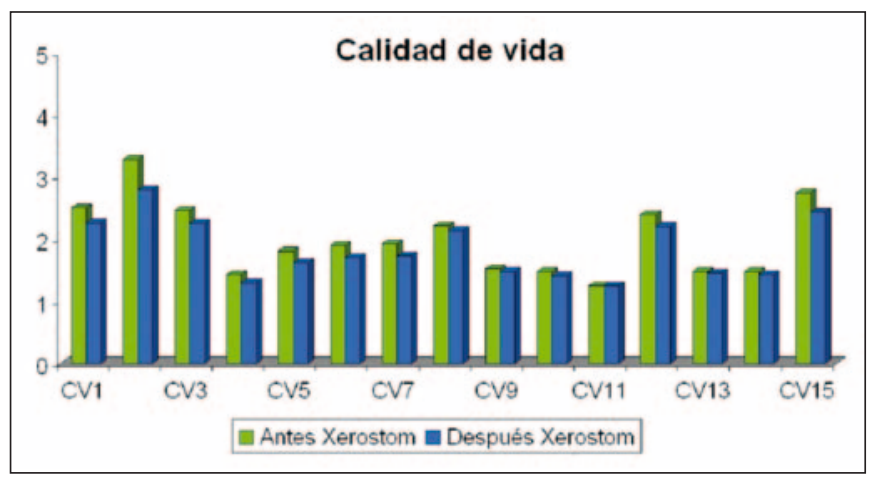

Fig. 1. Calidad de vida en base a las diferentes cuestiones planteadas.

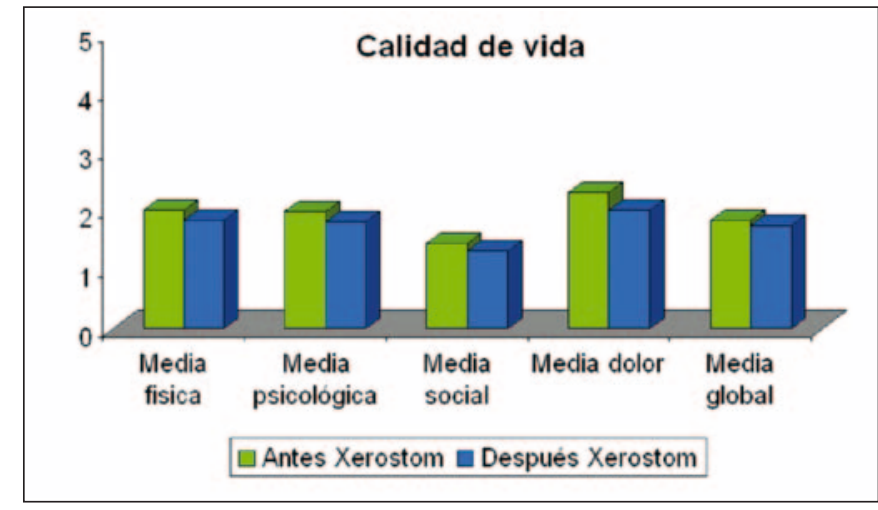

Fig. 2. Evaluación de diferentes parámetros relacionados con localidad de vida.

las preguntas (Figura 2): Función física (p: 0,0), función psicológica (p: 0,01), función social (p: 0,01) y dolor (p: 0,0).

\section{Escala visual analógica}

Hubo disminución en las puntuaciones de la escala visual analógica en todos los síntomas que evaluaba la escala visual analógica, después de la utilización de productos Xerostom ${ }^{\circledR}$. La reducción de síntomas fue estadísticamente significativa en 7 de los 8 síntomas (Figura 3), siendo la sequedad de garganta el único síntoma en el que las diferencias no fueron estadísticamente significativas. Especialmente importantes fueron la reducción en la sensación de sequedad de boca, y de lengua que mejoraron en el $75 \%$ de los pacientes, así como en el grado de sed, con un $57 \%$ paciente que mejoraron.

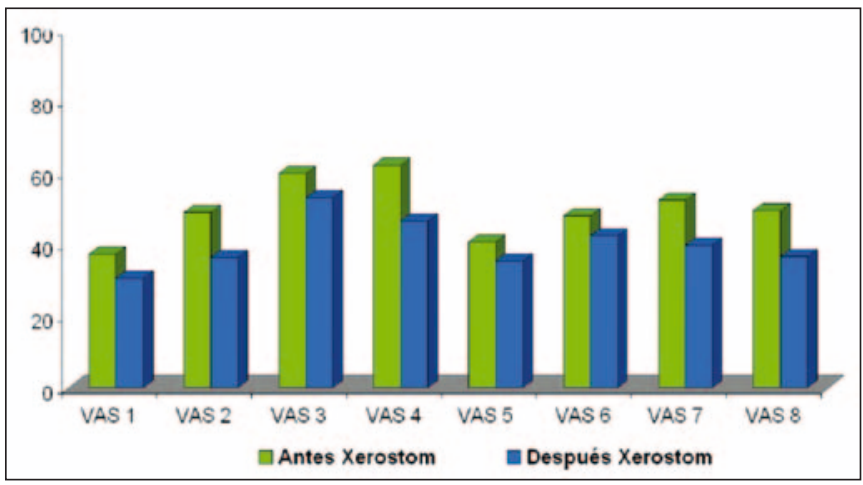

Fig. 3. Diferentes parámetros de la escasa visual analógica. 


\section{Flujo salivar}

El flujo salivar (Figura 4) no se pudo medir en 20 pacientes, en 18 de ellos, porque no presentaban ningún flujo salivar sin estimulación, y en 2 de ellos porque tras las cirugías orales realizadas tenían una imposibilidad mecánica para recoger la saliva. De los otros 20 pacientes, 4 tenían una medida flujo salivar normal, pero el aspecto de la saliva era muy viscoso y los pacientes tenían síntomas de xerostomía por tanto, aunque no se analizó la composición de la saliva, se trataba de una saliva alterada, incapaz de cumplir su función normal. La media de flujo salivar de todos los pacientes fue de 0,17 pero si excluimos a los 4 pacientes anteriormente referidos, fue de 0,09 . Tras la utilización de los productos Xerostom, mejoró el flujo salivar en 9 (45\%) de los 20 pacientes en los que se pudo medir. De estos 9 pacientes, 3 de ellos tuvieron una mejoría importante y la disminución de flujo salivar pasó de ser grado 2 a ser grado 1. La media del flujo salivar de todos los pacientes fue de 0,25 , excluyendo a los 4 pacientes anteriormente referidos fue de 0,14. Estas diferencias no fueron estadísticamente significativas.

\section{Efectos secundarios}

Ningún paciente refirió ningún efecto secundario importante o que le obligara a suspender la medicación. Solo un $7 \%$ refirió un escozor inicial rápidamente pasajero.

\section{DISCUSIÓN}

La xerostomía es un efecto secundario muy frecuente y con alto impacto en la calidad de vida de los pacientes tras el tratamiento radioterápico. Va a condicionar además otra serie de problemas que afectan no solo a la esfera física sino también a la psicológica y social.

Otro estudio (13) había demostrado que la utilización de productos basados en aceite de oliva, xilitol y betaína mejora los síntomas producidos por la xerostomía farmacológica. En el estudio que presentamos, vemos que también son útiles en pacientes con xerostomía tras irradiación.

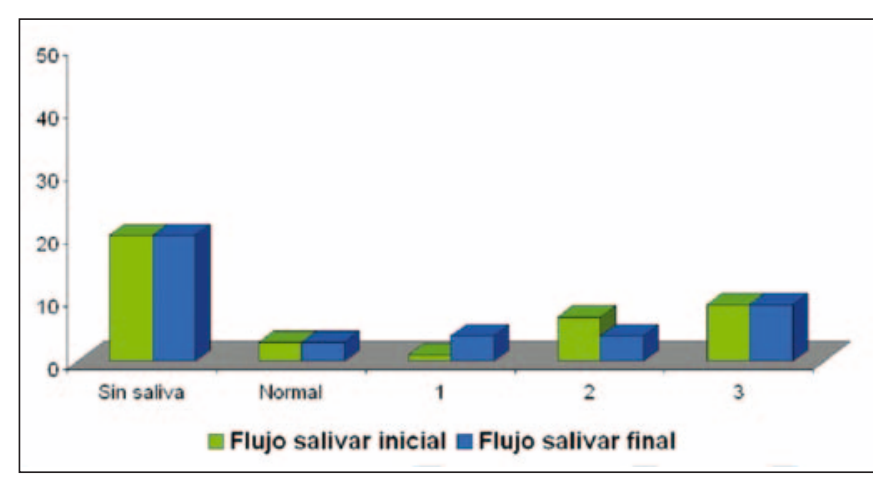

Fig. 4. Medición del flujo salival.

En lo que se refiere a la calidad de vida, las puntuaciones a la encuesta inicial, son sorprendentemente altas, teniendo en cuenta que los pacientes tenían un flujo salivar no estimulado muy bajo e incluso ausente. Sólo en la pregunta número 2, la sequedad de boca me resulta incómoda, la media está cercana al 4 de puntuación (correspondiente a la respuesta mucho), pero en el resto de preguntas apenas alcanza el 3 (correspondiente a la respuesta bastante). Esto puede ser debido a que como decíamos al principio algunos pacientes fueron reclutados tras conocer el resultado positivo de sus pruebas de revisión y tras saber que no había evidencia de recidiva de su enfermedad, lo que podría hacer que la xerostomía en ese momento les pareciera un problema menor. A pesar de estas puntuaciones positivas, la utilización de productos Xerostom ${ }^{\circledR}$ consigue mejorar la mayoría de los aspectos, 7 de forma significativa y globalmente en las 4 funciones a las que se refieren las preguntas: social, física, psicológica y personal. En el estudio realizado con xerostomía de causa farmacológica, la mejoría estadísticamente significativa se consiguió en 4 de las preguntas y en 3 de las funciones, física, dolor y personal pero no en la social.

En lo que se refiere a las preguntas de la escala visual analógica, los resultados son aún más favorables tras la utilización de Xerostom ${ }^{\circledR}$, hasta un $75 \%$ refieren disminución de la sensación de sequedad de boca y lengua y hasta un $56 \%$ de la sensación de sed. Muy importante es este último hecho porque, aunque no estaba recogido en la escala utilizada, estos pacientes referían menos interrupciones durante el sueño debido a la sequedad de boca. 


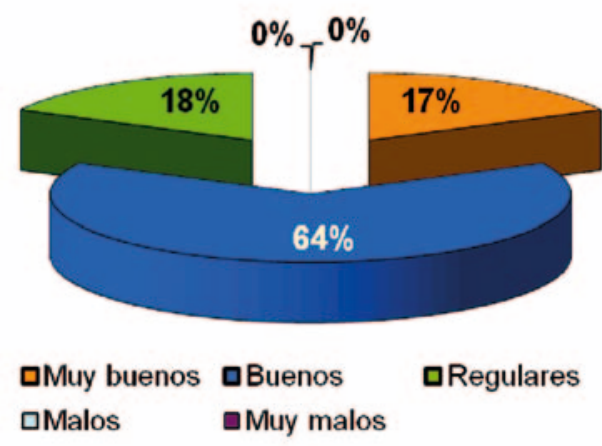

Fig. 5. Valoración de los diferentes productos utilizados.

El flujo salivar también mejoró, sorprendentemente, en un porcentaje alto de pacientes, indicando que tras el daño producido por la radioterapia, las glándulas salivares son capaces aún de funcionar bajo condiciones favorables.

Finalmente, el grado de satisfacción de los pacientes es muy alto. La gran mayoría de pacientes, el $82 \%$, refiere que los productos son buenos o muy buenos (Figura 5) y sólo un $8 \%$ los calificó como regulares. El producto mejor valorado fue el colutorio seguido por el spray y el gel (Figura 6).

\section{CONCLUSIONES}

La xerostomía es un problema muy prevalente y en los pacientes tratados con RT en el área ORL, que puede dar lugar a serias complicaciones tanto físicas como psicológicas. La prevención del daño a las glándulas salivares por la irradiación, es a veces imposible y las medidas farmacológicas para aumentar el flujo salivar, no están exentas de efectos secundarios.

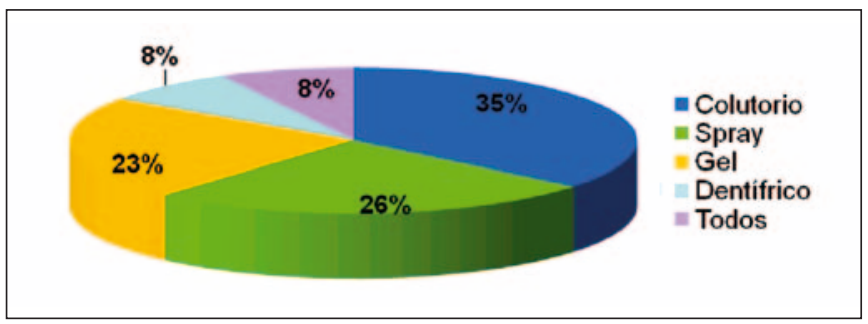

Fig. 6. Valoración de los diferentes productos en base a su presentación.
Sin embargo, medidas sencillas y sin efectos adversos, como la utilización de productos basados en aceite de oliva, betaína y xilitol, en forma de colutorio, pasta de dientes, gel y spray (Xerostom $\left.{ }^{\circledR}\right)$, mejora de forma importante la mayoría de los síntomas y la reducción de calidad de vida producidos por la boca seca. En algunos pacientes estos productos permiten, incluso, mejorar el flujo salivar.

Este estudio pone de relieve la importancia de mantener unos cuidados bucales adecuados, capaces no solo de evitar complicaciones sino de mejorar la xerostomía en pacientes con afectación seria de las glándulas salivares tras irradiación.

\section{BIBLIOGRAFÍA}

1. Eisbruch A, Dawson LA, Kim HM, Bradford CR, Terrell JE, Chepeha DB, et al. Conformal and intensity modulated irradiation of head and neck cancer: the potential for improved target irradiation, salivary gland function, and quality of life. Acta Otorhinolaryngol Belg 1999;53(3):2715.

2. Murdoch-Kinch CA, Kim HM, Vineberg KA, Ship JA, Eisbruch A. Dose-effect relationships for the submandibular salivary glands and implications for their sparing by intensity modulated radiotherapy. Int $J$ Radiat Oncol Biol Phys 2008 Oct 1;72(2):373-82.

3. Radfar L, Sirois DA. Structural and functional injury in minipig salivary glands following fractionated exposure to 70 Gy of ionizing radiation: an animal model for human radiation-induced salivary gland injury. Oral Surg Oral Med Oral Pathol Oral Radiol Endod 2003 Sep;96(3):267-74.

4. Almstahl A, Wikstrom M. Electrolytes in stimulated whole saliva in individuals with hyposalivation of different origins. Arch Oral Biol 2003 May;48(5): 337-44.

5. Kouloulias V, Thalassinou S, Platoni K, Zygogianni A, Kouvaris J, Antypas C, et al. The treatment outcome and radiation-induced toxicity for patients with head and neck carcinoma in the IMRT era: a 
systematic review with dosimetric and clinical parameters. Biomed Res Int 2013;2013:401261.

6. Sood AJ, Fox NF, O'Connell BP, Lovelace TL, Nguyen SA, Sharma AK, et al. Salivary gland transfer to prevent radiation-induced xerostomia: A systematic review and meta-analysis. Oral Oncol 2014 Feb;50(2):77-83.

7. Brizel DM, Wasserman TH, Henke M, Strnad V, Rudat V, Monnier A, et al. Phase III randomized trial of amifostine as a radioprotector in head and neck cancer. J Clin Oncol 2000 Oct 1;18(19): 3339-45.

8. Jellema AP, Slotman BJ, Muller MJ, Leemans CR, Smeele LE, Hoekman K, et al. Radiotherapy alone, versus radiotherapy with amifostine 3 times weekly, versus radiotherapy with amifostine 5 times weekly: A prospective randomized study in squamous cell head and neck cancer. Cancer 2006 Aug 1;107(3):544-53.

9. Le QT, Kim HE, Schneider CJ, Murakozy G, Skladowski K, Reinisch S, et al. Palifermin reduces severe mucositis in definitive chemoradiotherapy of locally advanced head and neck cancer: a randomized, placebo-controlled study. J Clin Oncol 2011 Jul 10;29(20):2808-14.

10. Abbasi F, Farhadi S, Esmaili M. Efficacy of pilocarpine and bromhexine in improving radioherapyinduced xerostomia. Dental Research, Dental Clinics, Dental Prospects 2012;7(2):86-90.

11. Witsell DL, Stinnett S, Chambers MS. Effectiveness of cevimeline to improve oral health in patients with postradiation xerostomia. Head Neck 2012 Aug;34(8):1136-42.

12. Furness S, Worthington HV, Bryan G, Birchenough S, McMillan R. Interventions for the management of dry mouth: topical therapies. Cochrane Database Syst Rev 2011;(12):CD008934.

13. Ship JA, McCutcheon JA, Spivakovsky S, Kerr AR. Safety and effectiveness of topical dry mouth products containing olive oil, betaine, and xylitol in reducing xerostomia for polypharmacy-induced dry mouth. J Oral Rehabil 2007 Oct;34(10):72432.

14. Pretty IA, Gallagher MJ, Martin MV, Edgar WM, Higham SM. A study to assess the effects of a new detergent-free, olive oil formulation dentifrice in vitro and in vivo. J Dent 2003 Jul;31(5):327-32.

15. Rantanen I, Nicander I, Jutila K, Ollmar S, Tenovuo $\mathrm{J}$, Soderling E. Betaine reduces the irritating effect of sodium lauryl sulfate on human oral mucosa in vivo. Acta Odontol Scand 2002 Oct;60(5):306-10.

16. Makinen KK, Makinen PL, Pape HR, Jr., Peldyak J, Hujoel P, Isotupa KP, et al. Conclusion and review of the Michigan Xylitol Programme (1986-1995) for the prevention of dental caries. Int Dent J 1996 Feb;46(1):22-34.

17. Pai S, Ghezzi EM, Ship JA. Development of a Visual Analogue Scale questionnaire for subjective assessment of salivary dysfunction. Oral Surg Oral Med Oral Pathol Oral Radiol Endod 2001 Mar;91 (3):311-6.

\section{CORRESPONDENCIA}

Dra. Margarita Martín Martín

Servicio de Oncología Radioterápica

Hospital de la Princesa

Diego de León, 62

28006 Madrid

Correo electrónico: margamm@yahoo.com 